\title{
Zur Kenntnis der Siedepunktsverhältnisse.
}

Von Richard Lonenz und W. Herz.

Vor einiger Zeit haben wir beide ${ }^{1}$ ) unabhängig voneinander auf SiedepunktsregelmäBigkeiten hingewiesen, wie sie zuerst in der bekannten Regel von GULDBERG-GOYE zum Ausdruck gekommen sind. Die Tatsache, daB sich seitdem eine ganze Anzahl von Forschern ${ }^{2}$ ) mit ähnlichen Betrachtungen befaßt haben, gibt uns Gelegenheit, gemeinsam einen weiteren Beitrag über die Siedepunktsverbältnisse za veröffentlichen.

Die Regel von Guldberg-Guye besagt, daß das Verhältnis des normalen Siedepunktes in absoluter Zählung zur ebenso gezählten kritischen Temperatur etwa zwei Drittel beträgt. Bei einem Briefwechsel zwischen uns wurde von R. LoRENz die Frage aufgeworfen, ob nicht vielleicht in der Skala der übereinstimmenden Temperaturen ein besserer Punkt herausgearbeitet werden könnte, als es der Siedepunkt ist, der bei verschiedenen Stoffen natürlich ganz verschiedenen Bruchteilen des kritischen Druckes entspricht. Es lag nahe, einen Vergleich der Siedetemperaturen bei gleichen Bruchteilen des kritischen Druckes durchzuführen, und W. Henz war in der Lage, aus seinen Berechnungen derartige Zusammenstellungen sofort zu liefern.

In den Landolt-Börnstein-Roth schen Tabellen sind zahlreiche Dampfdruckkurven angegeben, welche es gestatten, die zu gleichen Bruchteilen des kritischen Druckes gehörigen Siedetemperaturen zu interpolieren, und so entstand die nachfolgende Tafel, in der $T_{s 1}$ den Siedepunkt beim Druck von 1 Atmosphäre bedeutet und wobei gleichzeitig angegeben ist, welchen Bruchteil des kritischen Druckes $p_{k}$ eine Atmosphäre bedeutet. Unter $T_{s 2}$ stehen die Siedepunkte derselben Flüssigkeiten bei $1 / 33$ des kritischen Druckes und unter $T_{s 3}$ die Siedetemperaturen für $1 / 50$ des kritischen Druckes. In Klammern $108,253$.

1) W. Herz, diese Zeitschrift 91,$1 ; 95,253 ; 96,289$. R. Lorenz, ebenda

2) Jorissen, ebeada 104, 157; van LaAr, ebenda 104, $91 \mathrm{ff}$; van Lrempt, ebenda 111, 280; W ArDEN, ebenda 112, 225. 


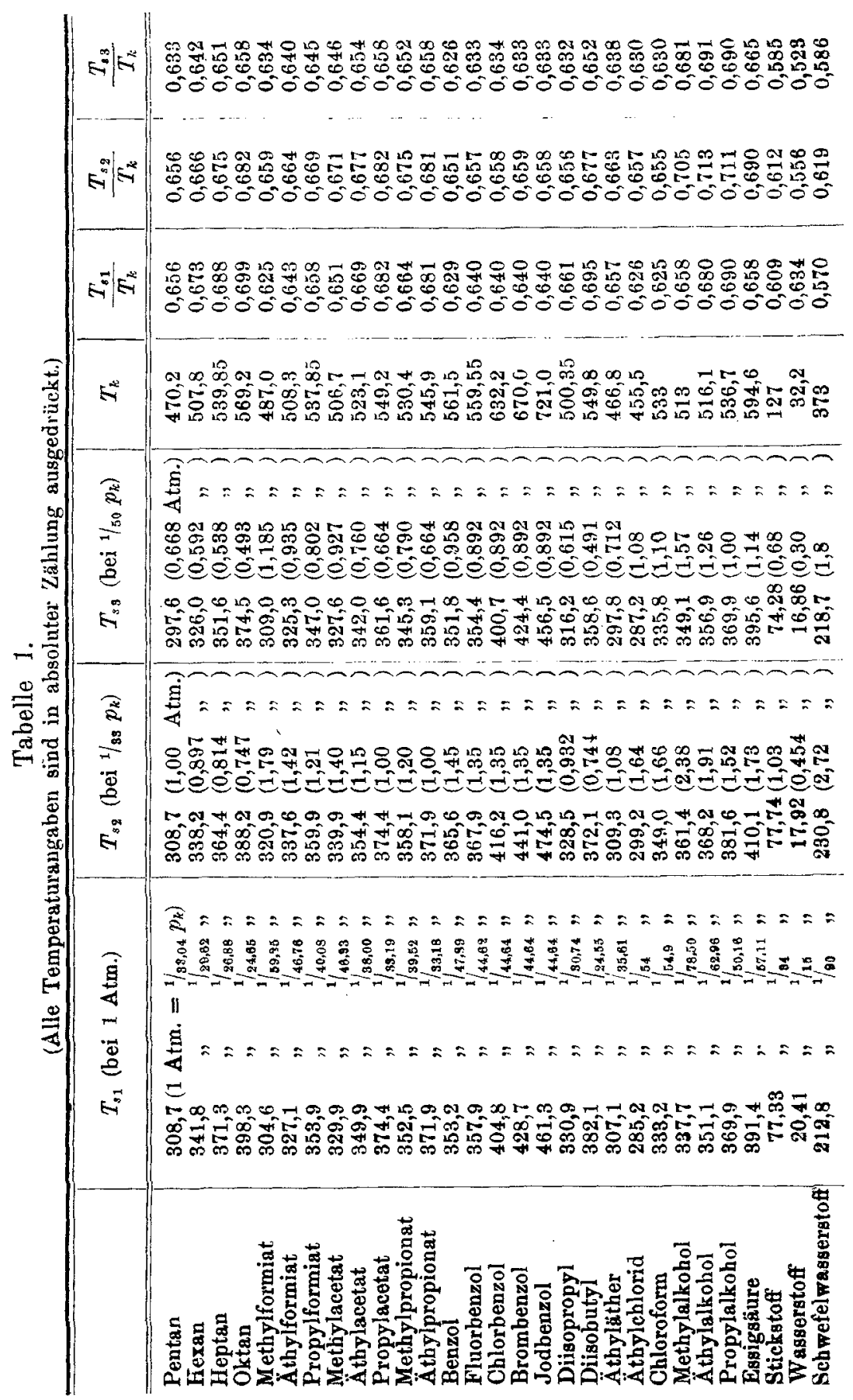


sind daneben die diesen Bruchteilen zugehörigen Atmosphären angegeben. Die Reihe unter $T_{k}$ liefert die kritischen Temperaturen, und die letzten drei Kolumnen zeigen die Quotienten dieser verschiedenen Siedetemperaturen und der kritischen Temperatur ${ }^{1}$ ) (s. Tabelle 1).

Bei einer oberflächlichen Betrachtung der Quotienten findet man, daB sie alle eine entschiedene Annäherung zur Konstanz zeigen, und man hat zunächst den Eindruck, daß es für die Genauigkeit der Verhältnisse gleichgültig ist, ob man die Siedetemperaturen bei 1 Atmosphäre, d. h. ungleichen Bruchteilen des kritischen Druckes in Beziehung zur kritischen Temperatur setzt, oder ob man solche Siedetemperaturen wählt, die zu gleichen Druckbruchteilen gehören. Eine schärfere Auswertung aber lehrt doch wohl, dab die Verhältnisse genauer werden, wenn man die Siedepunkte bei gleichen Bruchteilen des kritischen Druckes benutzt. Das zeigt die folgende Übersicht.

$$
T_{11}: T_{k}
$$

Höchster Wert 0,699 .

Tiefster Wert 0,570 .

\begin{tabular}{|c|c|c|c|c|}
\hline Zwischen & 0,560 & und & 0,579 & 1 \\
\hline 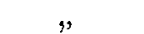 & 0,580 & $\eta$ & 0,599 & 0 \\
\hline "2 & 0,600 & 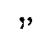 & 0,619 & 1 \\
\hline ", & 0,620 & " & 0,639 & 5 \\
\hline & 0,640 & "* & 0,659 & 11 \\
\hline & 0,660 & " & 0,679 & 4 \\
\hline " & 0,680 & $"$ & 0,699 & 7 \\
\hline
\end{tabular}

Zwischen 0,620 und 0,659 16 Fille ron 29.

$$
T_{s q}: T_{k}
$$

Höchster Wert 0,713 .

Tiefster Wert 0,556 .

$\begin{array}{cccccc}\text { Zwischen } & 0,556 & \text { und } & 0,579 & 1 & \text { Fall } \\ " & 0,580 & , & 0,599 & 0 & \\ " & 0,600 & " & 0,619 & \mathbf{2} & \text { Fülle } \\ " & 0,620 & " & 0,639 & 0 & " \\ " & 0,640 & " & 0,659 & 10 & " \\ " & 0,660 & " & 0,679 & 9 & " \\ " & 0,680 & " & 0,699 & 4 & " \\ " & 0,700 & " & 0,713 & 3 & \end{array}$

Zwischen 0,640 und 0,679 19 Fälle ron 29.

$$
T_{8 \mathrm{~s}}: T_{k} \text {. }
$$

Höchster Wert 0,691 .

Tiefster Wert 0,523 .

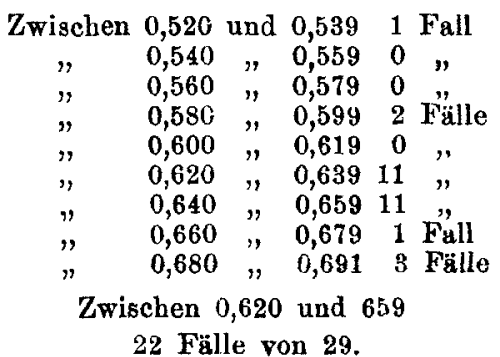

1) In bomologen Reihen organischer Verbindungen steigen die Quotienten mit wachsondem Kohlenstoffgehalt. 
Hier sind vom höchsten bis zum niedrigsten Quotientenwert immer in Abständen von 0,02 die Anzahlen der in jeden Abstand hineinfallenden Verhältnisse angegeben, und da zeigt sich, daB bei den normalen Siedepunkten innerhalb einer Differenz von 0,04 16 von den angeführten 29 Beobachtungen liegen, während in einen entsprechend großen Zwischenraum beim Vergleiche $T_{s 2}: T_{k} 19$ und bei $T_{s 3}: T_{k} 22$ Fälle kommen. Danach scheint also die Genauigkeit der konstanten Siedeverhältnisse am besten, wenn man die Siedetemperaturen bei $1 / 50$ des kritischen Druckes wählt. Allerdings ist die Gesamtdifferenz zwischen dem höchsten und viedrigsten Quotienten bei den normalen Siedepunkten kleiner als in den beiden anderen Fällen; doch haben natürlich einzelne Daten bei dieser Bewertung kein so großes Gewicht. Außerdem ist herrorzuheben, daB die überwiegende Zahl der geprüften Beispiele aus dem Gebiete der organischen Verbindungen stammt, bei denen die kritischen Daten nicht allzu verschieden sind und wo zwischen diesen und anderen Eigenschaften nahe einfache Beziehungen bestehen. ${ }^{1}$ ) Es wäre möglich, daB die Ergebnisse anders ausfallen würden, wenn genügende Beobachtungen bei zahlreicheren anorganischen Verbindungen mit ganz anderen kritischen Konstanten vorlägen; jedenfalls zeigen die Quotienten bei den von uns angeführten drei anorganischen Fällen eine entschiedene Tendenz zu kleineren Werten.

Schlieblich geben wir eine Tafel, in der die Siedepunkte bei gleichen Verhältnissen der kritischen Dichten zusammengestellt sind. $d_{k}$ und $T_{k}$ bedeuten kritische Dichte und kritische Temperatur. Der Siedepunkt $T$, welcher dem 21/2 fachen der kritischen Dichte zugehört, ist durch Interpolation aus den LandoLT-BöRNSTEIN-Rothschen Tabellen ermittelt.

Tabelle 2.

\begin{tabular}{|c|c|c|c|c|c|}
\hline & $d_{k}$ & $T_{k}$ & $21 / 2 \cdot d_{k}$ & $T$ & $\left.T: T_{k}^{2}\right)$ \\
\hline Methylacetat & 0,3252 & 506,7 & 0,8130 & 378,7 & 0,747 \\
\hline Methylpropionat & 0,3124 & 530,4 & 0,7810 & 395,8 & 0,746 \\
\hline Methylbutyrat & 0,3002 & 554,3 & 0,7505 & 416,4 & 0,752 \\
\hline Methylisobutyrat & 0,3012 & 540,55 & 0,7530 & 403,6 & 0,746 \\
\hline Äthylacetat & 0,3077 & 523,1 & 0,7692 & 392,4 & 0,750 \\
\hline Athylpropionat & 0,2965 & 545,9 & 0,7412 & 413 & 0,756 \\
\hline Propylformiat & 0,3093 & 537,85 & 0,7732 & 398,6 & 0,741 \\
\hline Propylacetat & 0,2957 & 549,2 & 0,7392 & 416,1 & 0,758 \\
\hline
\end{tabular}

1) Vgl. z. B. W. Hkrz, diese Zeitschrift 109, 293; 111, 52; 112, 278.

2) Auch dieser Quotient wächst in homologen organischen Reihen wenn auch nicht ausnahmslos - mit steigendem Molekelgewicht. 
104 R. Loren» u. W. Eori. Zur Kenntnis der Siedepunktsverhälnisse.

Tabelle 2 (Fortsetzung).

\begin{tabular}{|c|c|c|c|c|c|}
\hline & $d_{k}$ & $T_{k}$ & $21 / 2 \cdot d_{x}$ & $T$ & $T: T_{k}$ \\
\hline Methylalkohol & 0,2722 & 513 & 0,6805 & 400,3 & 0,780 \\
\hline Âthylalkohol & 0,2755 & 516,1 & 0,6887 & 395,8 & 0,767 \\
\hline Propylalkohol & 0,2734 & 536,7 & 0,6835 & 416,0 & 0,775 \\
\hline Pentan & 0,2323 & 470,2 & 0,5807 & 336,9 & 0.716 \\
\hline Hexan & 0,2344 & 507,8 & 0,5860 & 368,6 & 0,725 \\
\hline Heptan & 0,2341 & 539,85 & 0,5852 & 400,0 & 0,741 \\
\hline Oktan & 0,2327 & 569,2 & 0,5817 & 428,6 & 0,753 \\
\hline Isopentan & 0,2343 & 460,8 & 0,5857 & 325,1 & 0,705 \\
\hline Diisopropyl & 0,2411 & 500,35 & 0,6027 & 354,1 & 0,707 \\
\hline Diisobutyl & 0,2366 & 549,8 & 0,5915 & 468,75 & 0,844 \\
\hline Äthyläther & 0,2625 & 466,8 & 0,6562 & 340,6 & 0,730 \\
\hline Essigsäure & 0,3506 & 594,6 & 0,8765 & 437,7 & 0,736 \\
\hline Benzol & $0,304 \tilde{3}$ & 561,5 & 0,7612 & 399,4 & $0,71 \mathrm{l}$ \\
\hline Fluorbenzol & 0,3541 & 559,55 & 0,8852 & 400,1 & 0,715 \\
\hline Chlorbenzol & 0,3654 & 632,2 & 0,9135 & 459,7 & 0,727 \\
\hline Brombenzol & 0,4853 & 670,0 & 1,2132 & 487,5 & 0,728 \\
\hline Jodbenzol & 0,5814 & 721,0 & 1,4535 & 525,3 & 0,729 \\
\hline Kohlenstofftetrachlorid & 0,5576 & 556,15 & 1,3940 & 388,9 & 0,699 \\
\hline Sauerstoff & 0,4299 & 155 & 1,0747 & 102,1 & 0,659 \\
\hline Schwefeldioxyd & 0,52 & 430 & 1,30 & 321,6 & 0,748 \\
\hline Zinntetrachlorid & 0,7419 & 591,7 & 1,8547 & 430,7 & 0,730 \\
\hline
\end{tabular}

Die Quotienten $T: T_{k}$ zeigen gleichfalls eine Annäherung an einen konstanten Wert. Die Zahlen schwanken zwischen 0,844 und 0,659 ; docb liegen 18 von insgesamt 29 Fällen innerhalb 0,72 bis 0,76 .

Frankfurt a. M., Institut für physikalische Chemie der Universität und des physikalischen Vereins.

Breslau, Physikalisch-chemische Abteilung der Universität.

Bei dex Redaktion eingegangen am 11. Oktober 1920. 